\title{
Fall Perception for Elderly Care: A Fall Detection Algorithm in Smart Wristlet mHealth System
}

\author{
Zhinan $\mathrm{Li}^{1,2}$, Anpeng Huang ${ }^{1}, \mathrm{Wenyao} \mathrm{Xu}^{3}$, Wei $\mathrm{Hu}^{4}$, and Linzhen $\mathrm{Xie}^{1}$ \\ ${ }^{1} \mathrm{mHealth}$ Lab, School of EECS, Peking University, Beijing, China \\ ${ }^{2}$ Microsoft (China) Co., Ltd., Beijing, China \\ ${ }^{3}$ Department of Computer Science \& Engineering, SUNY Buffalo, NY, US \\ ${ }^{4}$ Wireless Healthcare Research and Development Center in Potevio, Beijing, China \\ \{lizhinan, hapku, tydxlz\}@pku.edu.cn, wenyaoxu@buffalo.edu, huwei@cpit.com.cn
}

\begin{abstract}
Mobile Health (mHealth) is expected to play a special role in today and the future healthcare delivery. Based on this trend, we design a Smart Wristlet mHealth system with mobile interface. The designed Smart Wristlet is dedicated to offer real-time alert for elderly fall, which is the most important when population ageing is becoming. In the Smart Wristlet mHealth system, fall detection is the "bottleneck" of the system operation. To remove this bottleneck away, we propose a fall perception solution for elderly care. In this proposal, we abstract and construct primitive-based features from raw data collected by the Smart Wristlet mHealth system, in which the most valuable features can be selected by using a TF-IDF (Term Frequency-Inverse Document Frequency) metric. In reality, these selected features are the most effective to perform fall detection. Our system tests and clinical trials demonstrate that this proposal is eligible to turn the Smart Wristlet mHealth system into a real solution for elderly care. Results show that the recognition precision and recall can reach $93 \%$ and $88 \%$, respectively. Compared with existing solutions, the gain from our proposal is an efficient prevention method for elderly fall, and can save more than 800 million dollars per year at today's socioeconomic level.
\end{abstract}

Keywords-mHealth (mobile Health), Smart Wristlet, Fall Perception, TF-IDF (Term Frequency-Inverse Document Frequency), Clinical Trials.

\section{INTRODUCTION}

A fall accident is the leading cause of accidental injury and death for the elderly people [1]. A hospital-based study found that $68 \%$ of elderly hospitalizations for injury are fall-related [2]. In addition, with a person getting older, he/she is at a higher risk to be involved in a fall injury. As reported in [2], for persons older than $85,86 \%$ of their injuries are from fall accidents. Only in United States, elderly falls cause around 8 billion dollars direct medical costs each year [3]. This huge loss has made elderly-fall be a major socio-economic problem. Thus, it is significant to prevent elderly-fall accidents. Recent advances in wireless mobile networking technologies have provided an opportunity to alleviate this problem. This concept is known as mobile health (mHealth) [4],[5],[6] which is changing health-care delivery today and is at the core of responsive health systems [4].
As observed above, a wearable system with mobile interfaces may play a vital role to detect falls and prevent elderly people from the related injuries [1],[3]. In such a system, sensor units (for example, accelerometers) are placed at human bodies to monitor user activities. In this system, the collected data streams are processed and analyzed in the embedded CPUs. In order to detect whether the wearer is falling, existing human activity recognition methods can be classified into four categories, namely, fixed threshold methods [7]; pattern recognition strategies [8]; conventional or fuzzy logic [9]; and artificial neural networks [10].

Unfortunately, these traditional methods are confronted with two main challenges. Firstly, their detection accuracy is relatively low, which cannot meet the requirements for elderly care and may cause serious medical negligence. Secondly, their high computing efficiency conflicts with terminal limitations in embedded wearable systems. This is because a highly complex computing process may easily drain the embedded CPU resources and the battery energy. In turn, the system response is also affected. This greatly restrains the service quality of elderly care because the fall detection accuracy, response time, and battery life are all the sensitive factors for an elderly fall prevention system.

In this study, we present a novel wearable sensor system called Smart Wristlet, which can provide 24 hours fall detection service. To ensure the reliability and practicability of Smart Wristlet, we developed a machine-perception based fall detection algorithm in it, which is called Fall Perception for short in the rest of this paper. Due to the high efficiency and accuracy of Fall Perception, Smart Wristlet achieves fall detection precision as high as $93 \%$, which is $3 \%$ higher than conventional methods. The recall is improved by $9 \%$ as well. More importantly, by simplifying the computational complexity and sensor data, the battery life is extended by more than $30 \%$. Obviously, these gains are very important to ensure long-time and uninterrupted mHealth services in real applications. For instance, with pop-up airbags on the user's body, Smart Wiristlet can trace user's activity and popup the airbags to protect him when a fall event is detected. Besides that, automate remote emergency alerting for elderly fall is also important since the old people usually are not able to call for help when they fall and get injured. 
The rest of the paper is organized as follows. In Section II, we will introduce our Smart Wristlet mHealth system first. Section III describes our Fall Perception mechanism. In Section IV, we will evaluate the performance of our proposal in clinical trials and system experiments. Finally, this study will be concluded in Section V.

\section{OVERVIW OF THE SMART WRISTLET MHEALTH SYSTEM}

For better understanding the devised Smart Wristlet mhealth system, we introduce the design requirements first. And then we present the wristlet mHealth system in terms of system architecture, hardware design, and system configuration.

\section{A. Design Requirements}

Smart Wristlet mHealth system is an innovative wearable sensor system designed for elderly care. Most importantly, Smart Wristlet is designed to sense users' activities in a noninvasive way, please see Fig. 1. This feature makes Smart Wristlet applicable for both inpatients and outpatients. In this mHealth system, sensors are embedded into a wristlet to acquire necessary user data. These embedded sensors are used to monitor movement parameters as well as physiological data. The system design and hardware basis turn the Smart Wristlet system to be a powerful tool for user activity analysis and health monitoring.

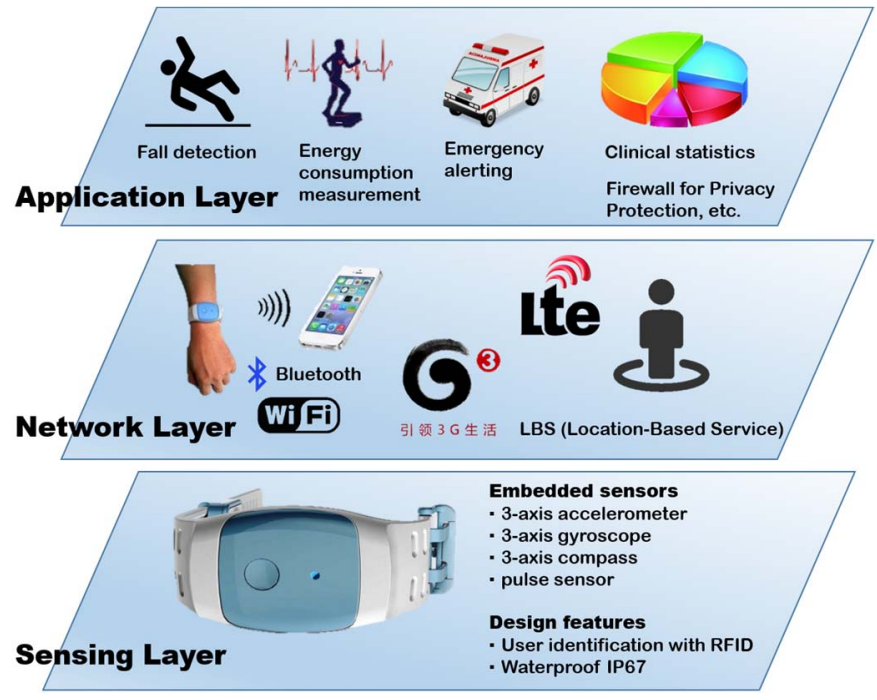

Fig. 1. The developed Smart Wristlet mHealth System (Contributions: Potevio developed the wristlet device, PKU conceived the fall perception algorithm and performed clinical trials).

\section{B. Architecture and Hardware}

The Smart Wristlet mHealth system consists of 3-layer function architecture, as shown in Fig. 1. In the sensing layer, a Smart Wristlet device is developed to capture users' information. Figure 2 shows the printed circuit board (PCB) of the Smart Wristlet terminal devices. In the terminal devices, a 3 -axis accelerometer, a 3-axis gyroscope and a 3-axis compass are embedded and grouped together to acquire user motion data for activity analysis. In addition, a pulse sensor is

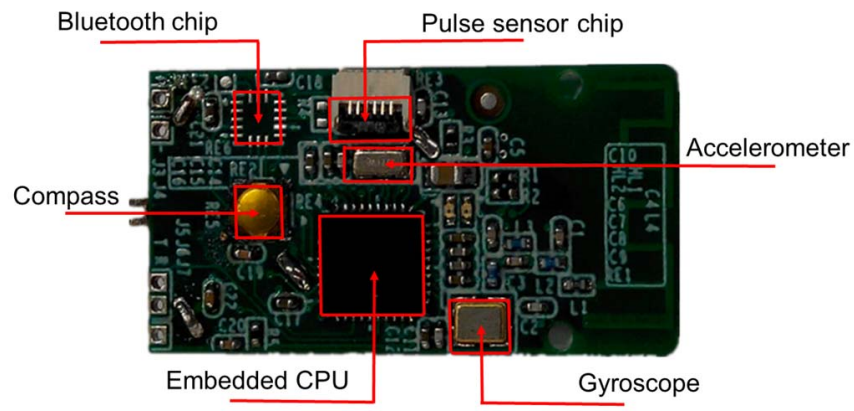

Fig. 2. Printed Circuit Board (PCB) of the Smart Wristlet device.

implanted to capture pulse signals. The pulse signals not only reflect health state, but also contain motion and mental information in Chinese traditional medical (which is left for further study in our project). A data reading chip and an embedded central processing unit (CPU) gather, process and analyze the acquired clinical data. This user device is powered by a $1200 \mathrm{mAh}$ Li-battery, and protected for waterproof in IP67 (for Ingress Protection, totally protected against dust at the toppest rate 6 , and protected against the effect of immersion between $15 \mathrm{~cm}$ and $1 \mathrm{~m}$ at the highest rate 7).

The network layer refers to the wireless data transmission between terminal devices and backend servers. After the acquired sensor data are processed and analyzed in the embedded CPU, the sensor data are sent to user smart phone via Bluetooth or $\mathrm{WiFi}$. Then, the data and alerting information can be virtualized on the smart phone, and sent to our database and alerting system for remote healthcare and emergency handling through $3 \mathrm{G}$ mobile networks. In this layer, user location-based service is also applied depending on the technology advances in mobile networks.

Based on the first two layers, many meaningful applications

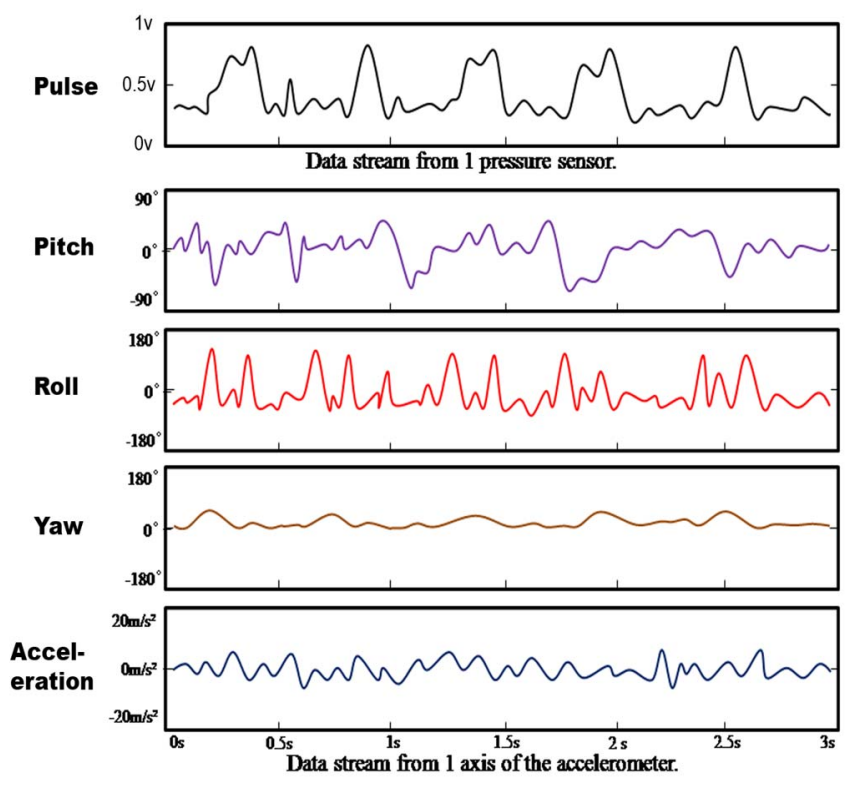

Fig.3. Data streams from Smart Wristlet sensors when walking straightly. 
can be carried out on the application layer, e.g., fall detection, emergency alerting and handling, pulse clinical statistics tracking, etc. In this paper, we focus on the enabling algorithm of fall detection techniques. In this layer, we also manage user accounts by using RFID in the Wristlet device. In this Smart Wristlet mHealth system, a firewall is also deployed for privacy data protection.

\section{System Implementation}

The sensors of Smart Wristlet can be divided into two types, the pulse and the motion sensors. The pulse sensor is to collect the pulse data for clinical decision-making support. A 3-axis accelerometer, a 3-axis gyroscope and a 3-axis compass are grouped as motion sensors. Data from the three motion sensors are used to recover the orientation (or gesture) of the wristlet, which are expressed by Roll angular, Pitch angular and Yaw angular (discussion of this orientation recovery work is out of this study scope, which will be further studied in the future).

Figure 3 shows the data streams from Smart Wristlet sensors. Smart Wristlet collects data that reflect the wearer's activity from multiple channels, i.e., pulse sensor, 3 orientation angles and 3-axis acceleration signals. Of course, these collected data streams are the input of an algorithm to perform real-time fall detection. Below, we explain how to conceive such an algorithm for fall detection.

\section{FALl Detection MEChanism}

To facilitate the Smart Wristlet mHealth system, we conceive a Fall Perception mechanism. In this study, the Fall Perception is a supervised framework, in which training data are mined offline in order to obtain a powerful data classifier. This derived classifier can make online pipeline be implemented efficiently. To achieve this goal, the Fall Perception is designed with two main features as below.

1. Time-sequential feature construction: Fall Perception constructs data features with time-sequential information, which can avoid the drawbacks of traditional primitivebased user motion analyzing approaches [11]. Most significantly, this approach can transform continuous raw sensor data into sparse symbols known as primitives, which simplifies the data structure. Additionally, the simple linear SVM can be applied to distinguish user motions for further simplifying computing complexity. As a result, Fall Perception can obtain higher efficiency without lowering accuracy requirement.

2. Primitive-based feature cleansing: Fall Perception cleanses the primitive features by analyzing their relevance to the focused motion. In the proposal, TF-IDF (Term Frequency-Inverse Document Frequency) [12] is used as a metric to select the relevant features from collected raw data, while the irrelevant features and misleading features are removed. This primitive feature cleansing procedure is capable of getting higher accuracy while reducing the dimensionality of the feature space for the concern of lower complexity.

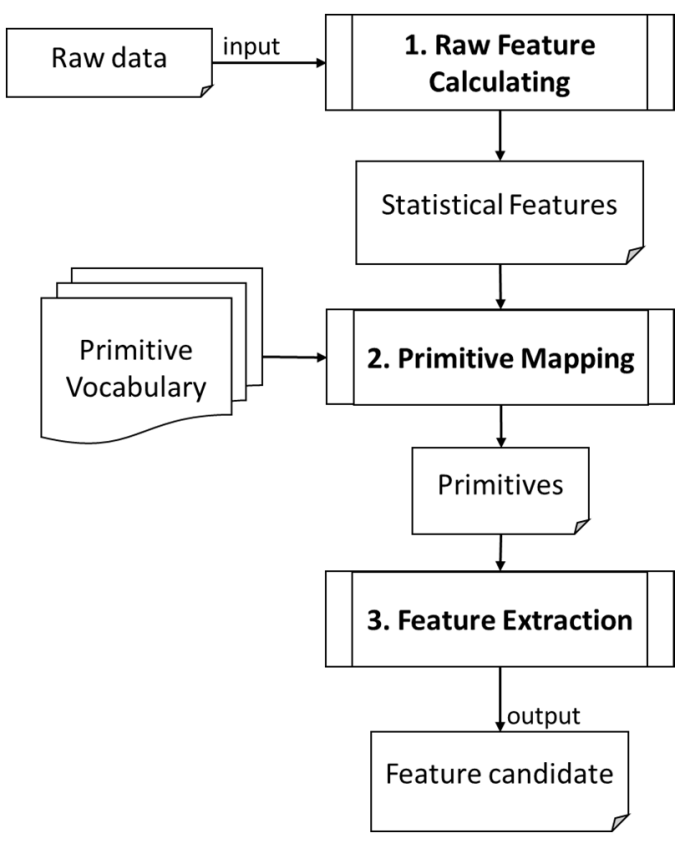

Fig.4. Movement-related data streams from the Smart Wristlet sensors.

Usually, these two metrics interact in a contradictory fashion. In the Smart Wristlet, Fall Perception achieves dramatic gain in both metrics using special designs, including primitive feature, TF-IDF feature selection, etc. In the following part, we elaborate the details of our Fall Perception from data preprocessing, feature selection and classifier training.

\section{A. Data Preprocessing}

Due to high complexity of Smart Wristlet raw data, they cause high burden to terminal batteries and CPUs. To solve this problem, the raw data need to be simplified. Fall Perception simplifies raw sensor data by transforming them to sparse symbols, called primitives [11]. The primitive transmission process works in a 2 -step process, please see step 1 and step 2 in Fig. 4. We use 6 statistical features to represent raw data information, including arithmetic mean, standard deviation, derivative mean, derivative variance, correlation mean and correlation variance. It is easy to understand that the number of feature combination is finite, we map each combination to one primitive. This mapping relation is called primitive vocabulary.

\section{B. Feature Extraction}

One drawback of current primitive based methods is that they lack consideration of the primitive orders or timesequential information [11]. This definitely causes information loss to the data mining computing. To overcome this drawback, the Fall Perception framework is to construct time-sequential features of primitives (Step 3 in Fig. 4). For this goal, we extend $\mathrm{N}$-grams theory [13] into the primitive feature extraction in this study. An $N$-gram is a contiguous sequence of $N$ items from a given sequence of primitives. For example, the primitive sequence "DZDCKDBC" contains 1-grams: D,Z,D,C,K,D,B,C; 2-grams: DZ,ZD,DC,CK,KD,DB,BC; and 3-grams: DZD, ZDC, DCK, CKD, KDB, DBC. There is a tradeoff in the determination of size $N$. If the value of $N$ is 
larger, the computing complexity is becoming higher. If $N$ is getting smaller, more information would be lost. How to determine the size of $N$ will be investigated in the future. In this way, the $\mathrm{N}$-grams of primitives are constructed and they are treated as feature candidates.

\section{Feature Selection}

In general, the number of obtained $N$-grams feature candidates is large. This phenomenon leads high computing overhead to online fall detection in the Smart Wristlet. In addition, some $N$-grams may be irrelevant or even misleading features. These misleading features are dangerous and may decrease the detection accuracy, especially when the training data are not clean and correct. To solve these concerns, the $\mathrm{N}$ gram features should be filtered to select proper ones.

The basic principle of filtering features is to select relevant features while discarding the irrelevant and misleading features. In the Fall Perception, three metrics are taken into account for evaluating the relevance of a feature $\mathrm{N}$-gram .

1) Occurrence Number of $N$-gram : Occurrence Number $^{-}$ of $\mathrm{N}-$ grami $_{i}$ is the number of $\mathrm{N}$-grami in positive training data sets. This metric is effective because common features tend to be relevant, while the rare features are usually tail or caused by noise.

2) Frequency of $N$-grami: Frequency of $N$-gram $i$ is the Occurrence Number of $\mathrm{N}$-grami divided by all $\mathrm{N}$ grams in the positive training data sets. Compared with Occurrence Number, Frequency can reduce the influence of training data set size.

3) TF-IDF of $N$-gram $_{i}$ : TF-IDF is a widely cited technique in text mining area, and it's applied to estimate the relevance between word terms and articles [12]. In this study, we define each $N$-gram as a term, and develop TF-IDF to evaluate the relevance between one $N$-gram and the positive training data set.

Based on these metrics, best features can be searched by fixed number. For example, we sort the $N$-grams ascendingly by their metric values, and define the first $K^{\text {th }}$ ones as relevant features. The algorithm performance with different $K$ values will be discussed in Section IV.

\section{Classifier Training}

In this step, the Fall Perception will judge whether the Smart Wristlet wearer is falling or not. Firstly, each training data unit can be mapped into a feature vector (with selected features). According to Step 3, the dimensionality of the feature vector is $3 \times K$. Although the data are high-dimensional, the feature vectors are usually sparse, and most of their elements are zero. To classify these data, linear SVM (Support Vector Machine) [14] is an efficient and effective tool. With the training data feature vectors, a binary linear SVM classifier is trained offline, which is essentially a $(3 \times K)$-length first order polynomial.

Then, a discrimination threshold is needed to distinguish the output of the classifier. If the output of the classifier is bigger than the threshold, the wearer will be regarded as falling, and vice versa. If a higher threshold is set, the detection precision can be improved. However, it is inevitable to lower down the rate of recall. This is because the precision and the recall are working at a conflict fashion. In reality, the threshold should be customized according to the application scenario.

Below, we perform system tests and clinical trials to verify effectiveness of this proposal.

\section{EXPERIMENTS AND EVALUATION}

To evaluate the performance of the Fall Perception framework, we conduct experiments in the Smart Wristlet system. In our study, 246 aging people in Beijing are chosen as experimental subjects. These subjects are all between 59 and 63 years old, and each of subjects performed standing, walking and falling motions wearing the Smart Wristlet. In system implementation, the TF-IDF metric is adopted to select $K$ proper $N$-gram features. The Smart Wristlet performance is tested in two major aspects - fall detection accuracy and battery life. For comparison in terms of fall detection, the conventional pattern recognition [15] and neural networks [16] are tested in the Smart Wristlet as well. The test performances are demonstrated below.

\section{A. Fall Detection Accurancy}

Firstly, we discuss the fall detection accuracy. Generally, the fall detection accuracy is quantified by precision and recall. (In this study, the precision and recall are the common concepts in data mining area.) Here,

$$
\begin{aligned}
& \text { Recall }=\frac{\{\text { Detected Fall Events }\}}{\{\text { Fall Events }\}}, \\
& \text { and Precision }=\frac{\{\text { Detected Fall Events }\} \cap\{\text { Fall Events }\}}{\{\text { Detected Fall Events }\}} .
\end{aligned}
$$

As discussed in Sec. III, the accuracy of the Fall Perception framework is affected by $K$-value of feature selection. For a larger $K$-value, more $N$-grams features will be selected and more variations of positive data will be contained. This is nature to improve fall detection accuracy because of more positive effect accumulated. But if the $K$-value is too large, some of unexpected features may be also involved into the classifier training, which affects the classifier performance. We

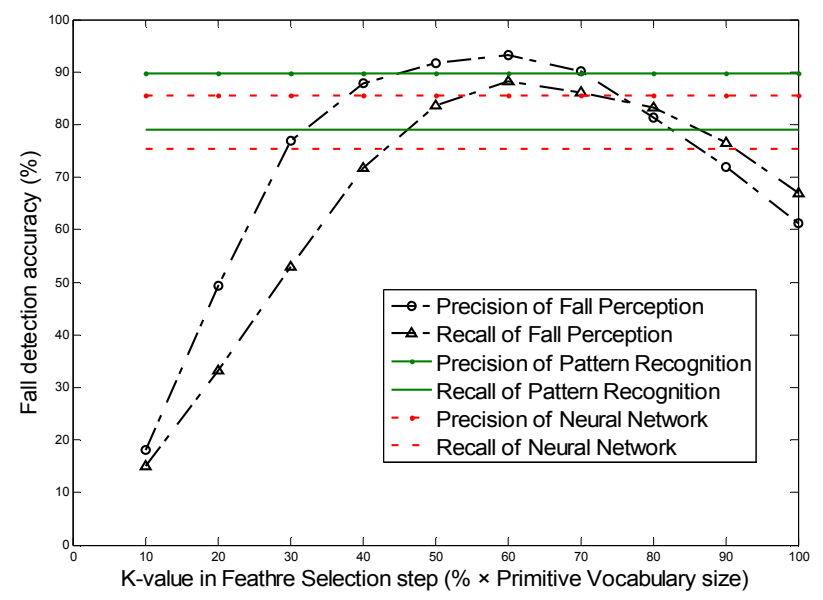

Fig. 5. Accuracy comparison with varying $K$-values. 
evaluated the algorithm accuracy with different $K$-values and the result is demonstrated in Figure 5. To consider the effect of $N$-gram feature set size, the $K$-value is quantized by comparison with the number of all $N$-gram features. As shown in Fig. 5, when $K$-value is about $60 \%$ of the $N$-gram feature set size, the precision and the recall reach their maximum value. Results show that the recognition precision and the recall can be improved $3 \%$ and $9 \%$, respectively, compared with exiting methods.

\section{B. Terminal Battery Life}

In terms of battery life, it is the basis for stable service of a wearable system. Clearly, to reduce power consumption, it is significant to minimize online computing complexity for extending battery life. Fall Perception achieves this goal by using sparse primitive features, selecting only top $K$ relevant features, and utilizing simple linear SVM classifier. Figure 6 shows the battery life of Smart Wristlet when it is employed with different fall detection algorithms. In this figure, the size of feature set is 600 . The experimental results show a lower power consumption of our proposal than those of traditional methods. To achieve the highest accuracy, $K$-value is set at $60 \%$ of the $N$-gram feature set size, which is equal to 360 . Then our proposal is able to extend the Smart Wristlet battery life to more than 24 hours, which is 6 hours longer than neural network method and 9 hours longer than pattern recognition methods. This feature of our proposal not only improves user experience of the Smart Wristlet, it also lowers the possibility of unexpected system breakdown and related elderly hurt.

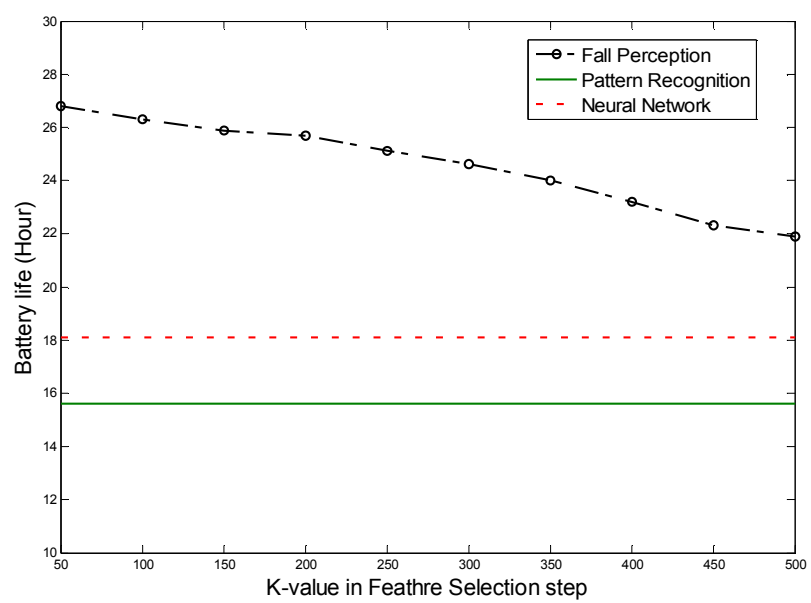

Fig. 6.Battery life of Smart Wristlet.

\section{CONCLUSION}

In this study, we propose a new fall detection solution based on machine-perception, called Fall Perception, which is a combination of efficiency and accuracy requirements. We perform system experiments and clinical trials in the developed Smart Wristlet mHealth system. Test results verify that this proposal is an effective mHealth tool to prevent elderly falls. Specifically, the fall detection accuracy can be improved, in which the performance gain can save more than 800 million dollars direct medical costs per year in the United States. Meanwhile, this proposal is helpful to turn the Smart Wristlet into real applications with required terminal performances, for example, longer battery life. More valuably, this proposal can be deployed to any similar mHealth systems if they need the fall detection.

\section{REFERENCES}

[1] T.Tamura, T. Yoshimura, M. Sekine, M. Uchida and O. Tanaka, "A Wearable Airbag to Prevent Fall Injuries," IEEE Transactions on Information Technology in Biomedicine, vol. 13, pp. 910 - 914, Nov. 2009 .

[2] C. DL, M. JG and C. TV, "Hospital resources used to treat theinjured elderly at North Carolina trauma centers," JAm Geriatr Soc. vol. 41, pp. 847-852, 1993.

[3] N. V. Carroll, P. W. Slattum, and F. M. Cox, "The Cost o f Falls Among the Community-Dwelling Elderly," Journal of Managed Care Pharmacy, JMCP, vol. 11, no. 4, May 2005.

[4] Global survey report, mHealth: New horizons for health through mobile technologies, www.who.int/goe/publications/goemhealthweb.pdf, World Health Organization, 2011.

[5] A. Huang, W. Xu, Z. Li, L. Xie and M. Sarrafzadeh, "System Lightloading Technology for mHealth: Manifold-Learning based Medical Data Cleansing and Clinical Trials in WE-CARE Project," IEEE Journal of Biomedical and Health Informatics, to be published.

[6] A. Huang, C. Chen, K. Bian, X. Duan, M. Chen, H. Gao, C. Meng, Q. Zheng, Y. Zhang, B. Jiao, and L. Xie, "WE-CARE: An intelligent mobile telecardiology system to enable mHealth applications," IEEE $J$. Biomedical Health Informatics, to be published.

[7] J. Fahrenberg, F. Foerster, M. Smeja and W. Muller, "Assessment of postureandmotion bymultichannel piezoresistive accelerometer recordings," Psychophysiol., vol. 34, pp. 607-612, 1997.

[8] P. H. Veltink, H. B. Bussmann, W. de Vries, W. L. Martens and R.C. van Lummel, "Detection of static and dynamic activities using uniaxialaccelerometers," IEEE Trans. Rehabil. Eng., vol. 4, no. 4, pp. 375-385,Dec. 1996.

[9] J. M. Winters, Y. Wang and J. M. Winters, "Wearable sensors and telerehabilitation," IEEE Eng. Med. Biol. Mag., vol. 22, no. 3, pp. 5665,May/Jun. 2003.

[10] K. Aminian, P. Robert, E. Je'quier and Y. Schutz, "Incline, speed, and distanceassessment during unconstrained walking," Med. Sci. Sports Exerc.,vol. 27, pp. 226-234, 1995.

[11] M. Zhang and A. A. Sawchuk, "Motion Primitive-Based Human Activity Recognition Using a Bag-of-Feature Approach," ACM SIGHIT International Health Informatics Symposium (IHI), Miami, Florida, USA, Jan. 2012.

[12] K. W. Church, W. A. Gale, "Inverse Document Frequency (IDF): A Measure of Deviations from Poisson," Natural language processing using very large corpora, Kluwer Academic Press, pp. 283-295, Boston, 1999.

[13] A. Brakensiek and G. Rigoll, "A comparison of character n-grams and dictionaries used for script recognition," Sixth International Conference on Document Analysis and Recognition, 2001. Proceedings., Seattle, WA, USA, 10-13 Sep. 2001.

[14] G.-X. Yuan and K.-L. Ma, "Scalable Training of Sparse Linear SVMs," IEEE 12th International Conference on Data Mining (ICDM), 2012, pp. 775-784, Brussels, Belgium, Dec. 10 - 13, 2012.

[15] C.W. Chang, and G.A.Bekey,"Pattern recognition applied to the prediction of post-operative gait in man,"1978 IEEE Conference on Decision and Control including the 17th Symposium on Adaptive Processes, pp. 787 - 788, San Diego, US, Jan. 1978.

[16] S.Zein-Sabatto, K.Kuah, R.Hajeomar and M.Bodruzzaman, "An intelligent vision system for motion detection using a neural network," Proceedings of the 1994 IEEE Southeastcon '94. Creative Technology Transfer - A Global Affair, pp. 362 - 366, 10-13 Apr. 1994. 